\title{
Medical Image Compression Using Wavelets And Vector Quantization For Telemedicine Application
}

\author{
Dr. A. A. Gurjar ${ }^{1}$ Neha S. Korde ${ }^{2}$ \\ (Electronics \& Telecommunication, Sipna College Of Engineering \& Technology, Amravati /SGBAU, Amravati, \\ India) \\ (Electronics \& Telecommunication, Sipna College Of Engineering \& Technology Amravati /SGBAU,Amravati, \\ India)
}

\begin{abstract}
A fundamental goal of image compression is to reduce the bit rate for transmission or data storage while maintaining an acceptable fidelity or image quality. To improve the classical lossless compression of low efficiency, a method of image lossless compression with high efficiency is presented. In this paper. The image compression scheme contains wavelet transformation and vector quantization. Wavelets allow complex information such as music, speech, images and patterns to be decomposed into elementary forms at different positions and scales and subsequently reconstructed with high precision and The vector quantization algorithms reduces the transmission bit rate or storage. After analyzing and implementing Wavelet transform in lossless compression, a new method of combining vector quantization with wavelet transform to compress medical images is discussed. The result from this presentation will give the better quality of the image.
\end{abstract}

Keywords: Image Compression, Lossless Compression, Medical Images, Vector Quantization, Wavelet Transform.

\subsection{NEED OF IMAGE COMPRESSION:}

\section{Introduction}

Images contain large amounts of information that requires much storage space, large transmission bandwidths and long transmission times. Therefore it is advantageous to compress the image by storing only the essential information needed to reconstruct the image. An image can be thought of as a matrix of pixel (or intensity) values. In order to compress the image, redundancies must be exploited, for example, areas where there is little or no change between pixel values. Therefore images having large areas of uniform colour will have large redundancies, and conversely images that have frequent and large changes in colour will be less redundant and harder to compress.

There are two parts to the compression:

1. Find image data properties; grey-level histogram, image entropy, correlation functions etc..

2. Find an appropriate compression technique for an image of those properties.

\subsection{MEDICAL IMAGE COMPRESSION:}

Medical images are very important in the field of medicine. Every year, terabytes of medical image data are generated through advance imaging modalities such as magnetic resonance imaging (MRI), computed tomography (CT), digital subtraction angiography (DSA), positron emission tomography (PET), X-rays and many more recent techniques of medical imaging. But storing and transferring these huge voluminous data could be a tedious job. some of the medical images are shown in fig. 1 .

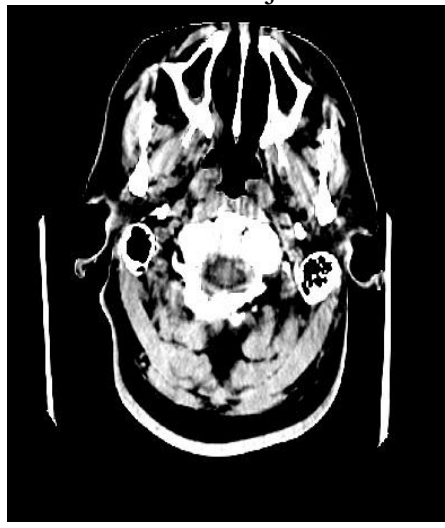

BRAIN IMAGE

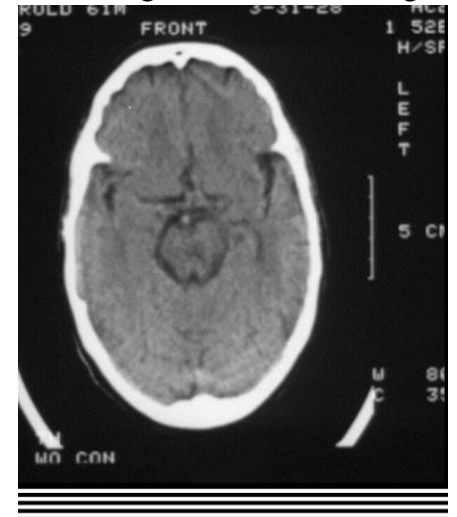

CT IMAGE OF BRAIN

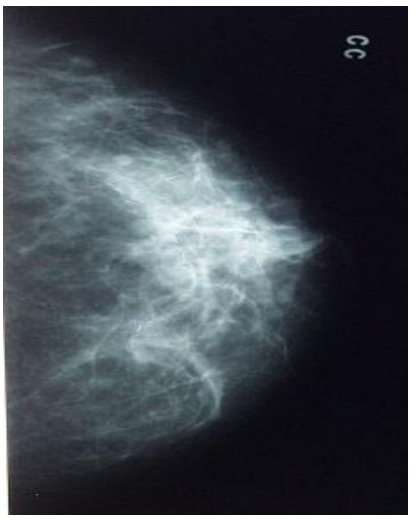

X-RAY IMAGE

Fig.1. Medical Images 
A compression of medical imagery is an important area of biomedical and telemedicine. In medical image compression diagnosis and analysis are doing well simply when compression techniques protect all the key image information needed for the storage and transmission. There are many medical image compression techniques are available and evolving in day to day basis. The study of all such compression techniques are important, different techniques uses different medical images like Magnetic resonance images (MRI) and X-ray angiograms (XA) etc. DICOM (digital imaging and communications in medicine) is used for storing, transmitting and viewing of the medical images.

\subsection{TELEMEDICINE}

Telemedicine is the use of telecommunication and information technologies in order to provide clinical health care at a distance. It is the use of medical information exchanged from one site to another via electronic communications. Telemedicine includes a growing variety of applications and services using two-way video, email, smart phones, wireless tools and other forms of telecommunications technology. The other popular technology is the two-way interactive television (IATV). This is used when a 'face-to-face' consultation between the health expert and the patients become mandatory. It is usually between the patients and their provider in one location and a specialist in another location.

Many potential benefits of telemedicine can be envisaged, including:

_ improved access to information;

_ provision of care not previously deliverable;

_ improved access to services and increasing care

delivery;

_ improved professional education;

_ quality control of screening programmes;

_ reduced health-care costs.

\subsection{EXISTING METHODS}

\section{Frame Work Of Our Proposed Method}

\subsubsection{Medical Image Compression Using Wavelets}

In this paper an attempt has been made to analyse different wavelet techniques for image compression. Hand designed wavelets considered in this work are Haar wavelet, Daubechie wavelet, Biorthognal wavelet, Demeyer wavelet, Coiflet wavelet and Symlet wavelet. These wavelet transforms are used to compress the test images competitively by using Set Partitioning In Hierarchical Trees (SPIHT) algorithm. SPIHT is a new advanced algorithm based on wavelet tra nsformwhich is gaining attention due to many potential commercial applications in the area of image compression. The SPIHT coder is also a highly refined version of the EZW algorithm.

\subsubsection{Medical Image Compression using Wavelet Decomposition for Prediction Method}

Method is based on wavelet decomposition of the medical images followed by the correlation analysis of coefficients. The correlation analyses are the basis of prediction equation for each sub band. Predictor variable selection is performed through coefficient graphic method to avoid multicolinearity problem and to achieve high prediction accuracy and compression rate. The method is applied on MRI and CT images. Two MRI and two CT gray scale standard test images as shown in figure 2 of size128*128 have been taken from world wide web for experiments and comparisons. MATLAB 7.0 has been used for the implementation of the proposed approach and results have been conducted on Pentium-1V, $3.20 \mathrm{GHz}$ processor with a memory of 512 MB. BPP (Bits Per Pixel) metric is evaluated to compile compression result. Every image was decomposed into three scales with 10 wavelet sub bands.

\subsubsection{ROI-based DICOM image compression for telemedicine}

Many classes of images contain spatial regions which are more important than other regions. For medical images, only a small portion of the image might be diagnostically useful, but the cost of a wrong interpretation is high. Hence, Region Based Coding (RBC) technique is significant for medical image compression and transmission. A CT or MRI image contains three parts, ROI (the diagnostically important part), Non-ROI image part, and the background (part other than image contents). The ROI is selected by expert radiologists. Depending on the selected part ROI-mask is generated in such a way that the foreground is totally included and the pixel values in the background are made zero. The background regions though they appear to be black in colour, they do not have zero grey level values. Algorithm is implemented on a group of MR DICOM images. SPIHT is proved to be the best. But for ROI-based compression computational complexity is also one of the important issues to be considered, while addressing real time applications. A new and simple 
algorithm as explained above is used to encode the image. Original image formatted in DICOM format of size $256 \mathrm{X} 256$ with 8 bit resolution is input to software. The „,compressed image ${ }^{e e}$ is the image which is generated at the decoder side after reconstruction process. The output of encoder is a bit stream of numbers arranged in a manner so as to support the progressive transmission, with initial part as a ROI compressed with run length encoding. This bit stream is transmitted over the telemedicine network using GSM mobile device.

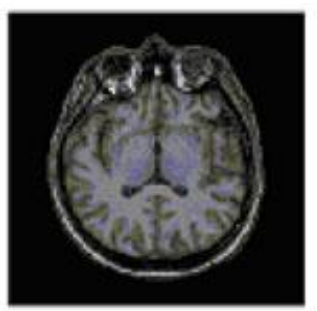

Original Image

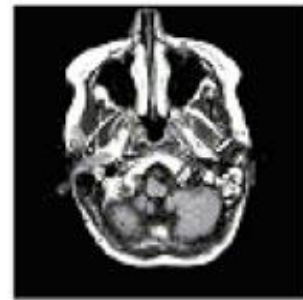

Original Image

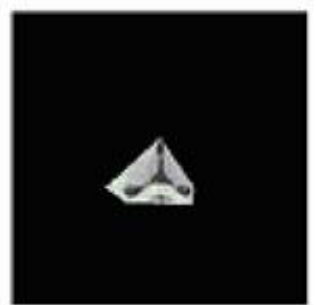

Region of Interest

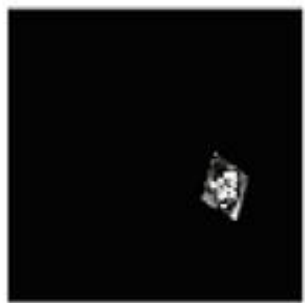

Region of Interest

Fig. 2.ROI-Based DICOM Image Compression

\subsection{PROPOSED METHOD}

In the proposed work wavelet transform and vector quantization are the tool which we are going to use for medical image compression After analyzing and implementing Wavelet transform in lossless compression, a new method of combining vector quantization with wavelet transform to compress medical images. Wavelet transform has some of the advantages over the traditional transforms like

1. More robust under transmission and decoding errors.

2. Better matched to the HVS characteristics.

3. Good frequency resolution at lower frequencies, good time resolution at higher frequencies - good for natural images.

\subsubsection{BASICS OF WAVELET TRANSFORM}

The wave is an infinite length continuous function in time. In contrast, wavelets are localized waves. A wavelet is a waveform of an effectively limited duration that has an average value of zero. Wavelet transform of a function is the improved version of Fourier transform. It provides the time-frequency representation. . The fundamental idea of wavelet transforms is that the transformation should allow only changes in time extension, but not shape. This is effected by choosing suitable basis functions that allow for this Changes in the time extension are expected to be conform to the corresponding analysis frequency of the basis function. Wavelet transforms are based on small wavelets with limited duration. The Continuous Wavelet Transform or CWT formally it is written as:

$\gamma(\mathrm{s}, \Gamma)=\int \mathrm{f}(\mathrm{t}) \psi^{*} \mathrm{~s}, \mathrm{r}(\mathrm{t}) \mathrm{dt}$

where * denotes complex conjugation. This equation shows how a function $f(t)$ is decomposed into a set of basis functions $\psi \mathrm{s},-(t)$, called the wavelets. The variables $s$ and - are the new dimensions, scale and translation, after the wavelet transform. equation (2) gives the inverse wavelet transform

$\mathrm{f}(\mathrm{t})=\iint \gamma(\mathrm{s}, \Gamma) \psi \mathrm{s}, \Gamma(\mathrm{t}) \mathrm{d} \Gamma \mathrm{ds}$

The wavelets are generated from a single basic wavelet $\psi(t)$, the so-called mother wavelet, by scaling and translation

$\Psi_{\mathrm{s}, \mathrm{r}}(\mathrm{t})=1 / \mathrm{N}_{\mathrm{s}} \psi(\mathrm{t}-\mathrm{r} / \mathrm{s})$

In (3) $s$ is the scale factor, - is the translation factor and the factor $s-1 / 2$ is for energy normalization across the different scales. 


\subsubsection{DISCRETE WAVELET TRANSFORM}

Discrete wavelets are not continuously scalable and translatable but can only be scaled and translated in discrete steps. This is achieved by modifying the wavelet representation (3) to create $\Psi_{\mathrm{j}, \mathrm{k}}(\mathrm{t})=1 /{ }_{\mathrm{s} 0 \mathrm{j}} \psi(\mathrm{t}-\mathrm{k} \mathrm{k} 0 \mathrm{~s} / \mathrm{s} \mathrm{s} 0 \mathrm{j})$

Although it is called a discrete wavelet, it normally is a (piecewise) continuous function. In (10) $j$ and $k$ are integers and $s 0>1$ is a fixed dilation step. The translation factor -0 depends on the dilation step. The effect of discretizing the wavelet is that the time-scale space is now sampled at discrete intervals. We usually choose $s 0=$ 2 so that the sampling of the frequency axis corresponds to dyadic sampling. This is a very natural choice for computers, the human ear and music for instance. For the translation factor we usually choose $-0=1$ so that we also have dyadic sampling of the time axis. In the analysis of both numerical and functional methodologies, a Discrete Wavelet Transform (DWT) can be used. DWT is a kind of wavelet transform for which the wavelet functions are discretely sampled by the other wavelet transforms. A major advantage of discrete wavelet transform over the Fourier transform is the effect of temporal resolution.

Different types of wavelets are given below these all types of wavelets are used for image compression for telemedicin application

1. Harr wavelet

2. Coiflet wavelet

3. Daubechies wavelet

4. Symlet

5. Demeyer

\subsubsection{VECTOR QUANTIZATION}

The most powerful and quantization technique used for the image compression is vector quantization(VQ).The vector quantization algorithms for reducing the transmission bit rate or storage have been extensively investigated for speech and image signals. Image vector quantization (VQ) includes four stages: vector formation,Training set selection, codebook generation and quantization. The first step is to divide the input image into set of vectors. The Subset of vectors in the set is later chosen as a training sequence. The codebook of codewords is obtained by an iterative clustering algorithm. Finally, in quantizing an input vector, closest codewords in the codebook is determined and corresponding label of this code word is transmitted. In this process, data compression is achieved because address transmission requires fewer bits than transmitting vector itself. The concept of data quantization is extended from scalar to vector data of arbitrary dimension. Instead of output levels, vector quantization employs a set of representation vectors (for one dimensional case) or matrices (for two dimensional cases). Set is defined as "codebook" and entries as "codewords". Vector quantization has been found to be an efficient coding technique due to its inherent ability to exploit the high correlation between the neighboring pixels.

\section{The Encoder}

The Decoder

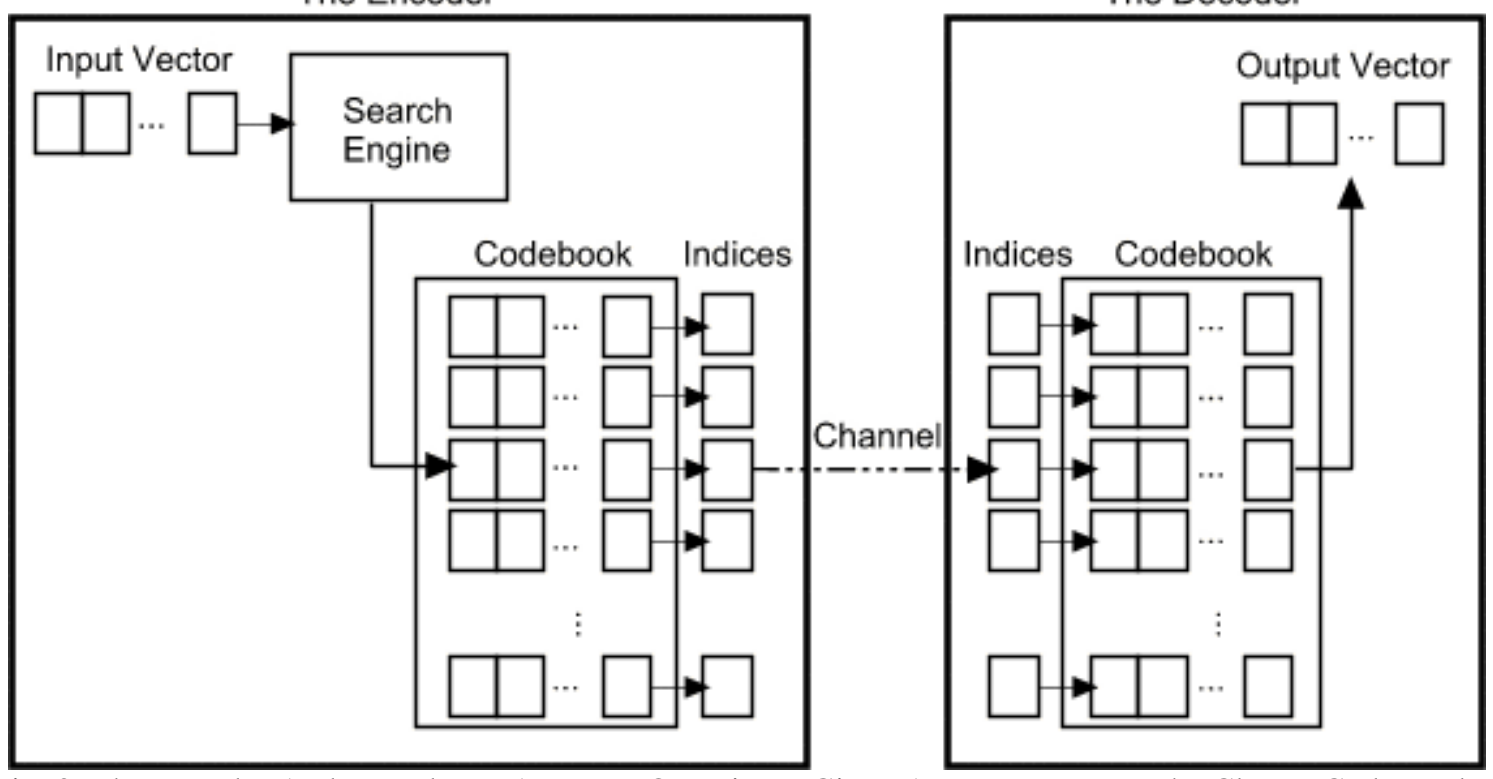

Fig. 3. The Encoder And Decoder In A Vector Quantizer. Given An Input Vector, The Closest Codeword Is Found And The Index Of The Codeword Is Sent Through The Channel. The Decoder Receives The Index Of The Codeword, And Outputs The Codeword. 


\subsubsection{DESIGN METRICS}

Digital image compression techniques are examined with various metrics. Among those the most important one is Peak Signal to Noise Ratio (PSNR) which will express the quality. There exists another property which expresses the quality, that is, Mean Square Error (MSE). PSNR is inversely proportiona 1 toMSE. The other important metric is Compression Ratio, which express the amount of compression embedded in the technique. In theory, it was observed that PSNR and Compression ratios are inversely related.

\subsubsection{Mean Square Error}

Among the quantitative measures, a class of criteria used often is called the mean square criteria. It refers to some sort of average or sum (or integral) of squares of the error between two images. .MSE for monochrome image

$$
1 / \mathrm{N}^{2} \sum_{\mathrm{I}}^{\mathrm{N}} \sum_{\mathrm{j}}^{\mathrm{M}}(\mathrm{X}(\mathrm{i}, \mathrm{j})-\mathrm{Y}(\mathrm{i}, \mathrm{j}))^{2}
$$

MSE for color image,

$$
1 / \mathrm{N}^{2} \sum_{\mathrm{i}}^{\mathrm{N}} \sum_{\mathrm{j}}^{\mathrm{N}}\left[\left(\mathrm{r}(\mathrm{i}, \mathrm{j})-\mathrm{r}^{*}(\mathrm{i}, \mathrm{j})\right)^{2}+(\mathrm{g}(\mathrm{i}, \mathrm{j})-\mathrm{g} *(\mathrm{i}, \mathrm{j}))^{2}+(\mathrm{b}(\mathrm{i}, \mathrm{j})-\mathrm{b} *(\mathrm{i}, \mathrm{j}))^{2}\right]
$$

where $r(i, j), g(i, j)$ and $b(i, j)$ represents a color pixel in location(i,j) of the original image, $r *(i, j), g *(i, j)$ and $b^{*}(\mathrm{i}, \mathrm{j})$ represents color pixel of the reconstructed image and $\mathrm{N} X \mathrm{~N}$ denotes the size of the pixels of these color images. $\sigma^{2}$ where is the variance of the desired image and $\sigma_{\mathrm{e}}^{2}$ is average variance.

\subsubsection{Peak Signal to Noise Ratio}

Peak Signal to Noise Ratio is defined as the ratio between signal variance and reconstruction error variance. Mean Square Error, Peak Signal to Noise Ratio and Compression Ratios are calculated from thefollowing expressions.

$\mathrm{PSNR}=10 \log _{10} 255_{\mathrm{MSE}^{2}}^{2}$

\subsubsection{Compression Ratio}

Compression ratio is defined as the ratio between the original image size and compressed image size. Compression Ratio $=$ size of Compressed image $/$ size of Original image

III. FIGURE

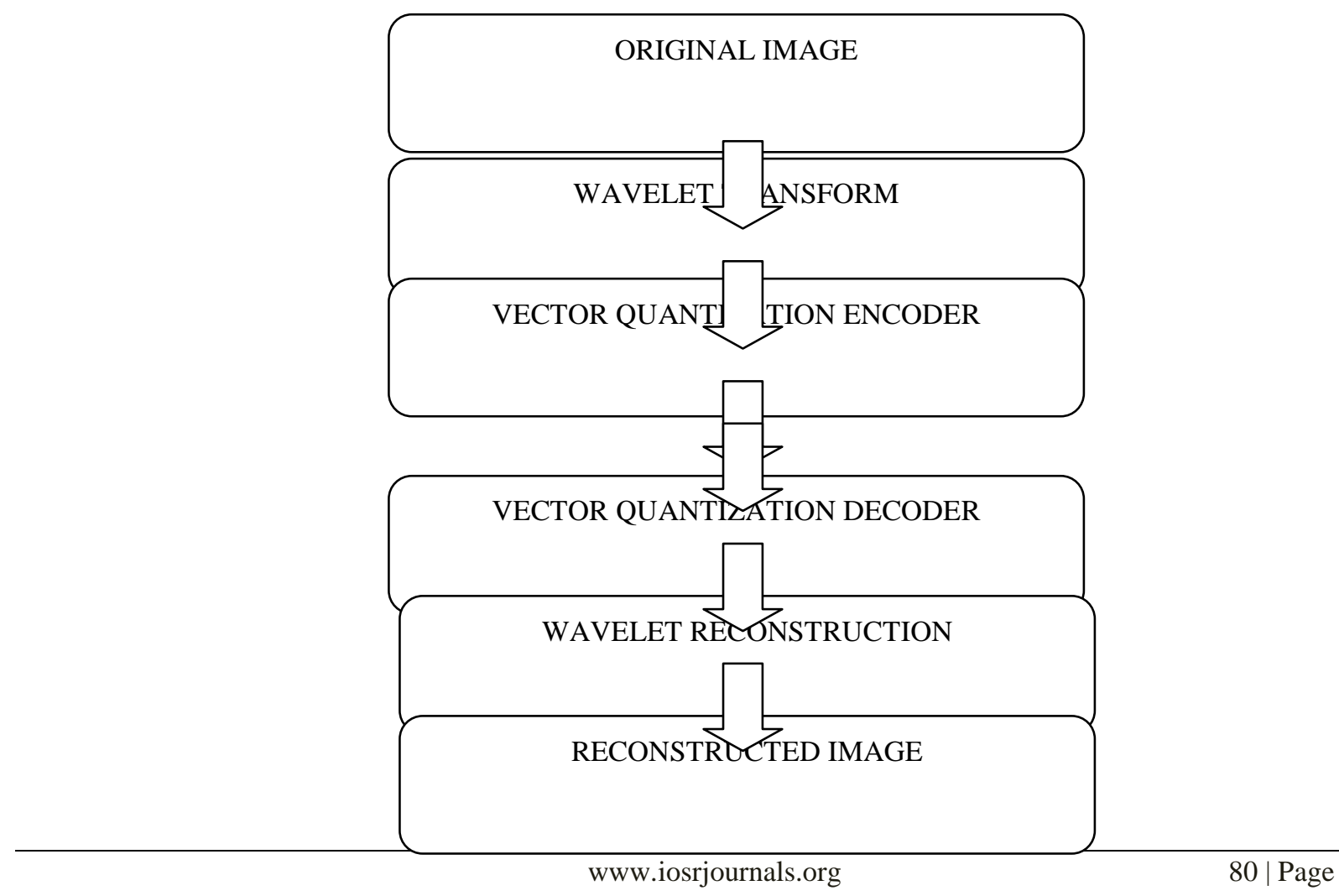


Fig. 4. Flow Diagram Of The Proposed Method.

IV. Simulation Results

In this section the simulation results of the said techniques are presented, which are obtained after implementing those in MATLB. The design metrics considered are already presented in the previous sections .The compression ratio should be maintained with considerable quality or in other way; PSNR should be maintained with sufficient size reduction in the memory point of view. The GUI used in the work was given in the figure 2. Wide range of medical images, including both MRI and CT scan images were considered. The algorithms are implemented in MATLAB. The GUI used in the work was given in the figure 1. The input mages are shown in figure 1 . The table 1 gives the performance of hand designed wavelet transform on the input images. The performance of these wavelet transforms was analyzed and plotted in figure 3

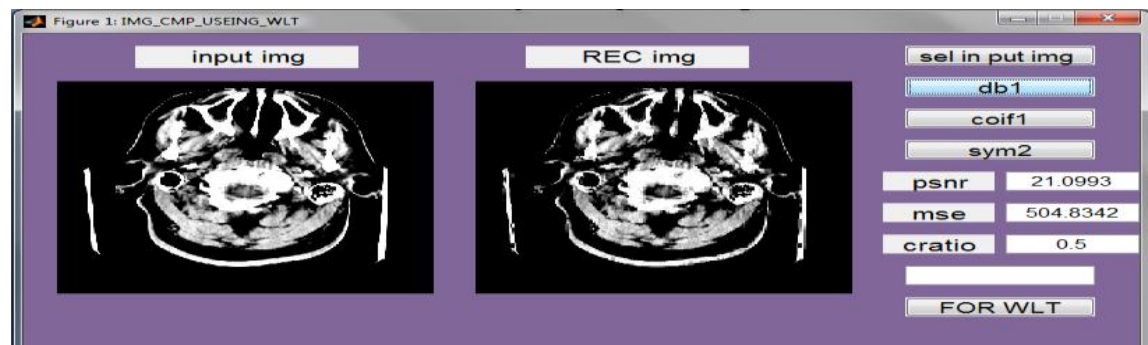

Fig.5. GUI Used In Matlab

Table 1: Comparison of PSNR, MSE \& CR USING DIFFERENT WAVELET

\begin{tabular}{|c|c|c|c|c|c|}
\hline Image & Parameter & Haar & Coiflet & Symlet & Demeyer \\
\hline \multirow[t]{2}{*}{ Brain Image } & PSNR(bpp) & 21.0993 & 24.4246 & 22.8399 & 24.5003 \\
\hline & $\operatorname{MSE}(\mathrm{db})$ & 504.83 & 234.7554 & 338.1338 & 230.7017 \\
\hline \multirow{2}{*}{$\begin{array}{l}\text { CT Image Of } \\
\text { Brain }\end{array}$} & PSNR(bpp) & 14.8971 & 19.4283 & 20.2823 & 23.7595 \\
\hline & $\operatorname{MSE}(\mathrm{db})$ & 2105.5626 & 741.7388 & 609.3239 & 273.6074 \\
\hline \multirow[t]{3}{*}{ X-Ray Image } & PSNR(bpp) & 35.135 & 35.9725 & 35.8121 & 36.2558 \\
\hline & $\operatorname{MSE}(\mathrm{db})$ & 19.9335 & 16.4372 & 17.0559 & 15.3992 \\
\hline & $\mathrm{CR}$ & 0.4988 & 0.47925 & 0.48361 & 0.21519 \\
\hline
\end{tabular}

\section{Conclusion:}

Medical image compression is the current research area of interest.In this paper, the performance of Hand designed wavelets is presented. Haar wavelet, Demeyer wavelet, Coiflet wavelet and Symlet wavelets are considered under Hand designed wavelet category. Hence this is more suitable for the transmission of Medical images for Telemedicine application. The experimental results show its concision, high compression ratio and practicability.

\section{Acknowledgements}

I am very much thankful to Dr.A.A.Gurjar sir, faculty of Electronics \& Telecommunication, Sipna COET, for their valuble guidance.

\section{Journal Papers:}

\section{References}

[1] Lihong ZHAO, Yanan TIAN, Yonggang SHA, Jinghua LI, Medical image lossless compression based on combining an integer wavelet transform with DPCM, Front. Electr. Electron. Eng. China 2009, 4(1): 1-4 DOI 10.1007/s11460-009-0014-1

[2] K. Somasundaram, and S.Domnic, Modified Vector Quantization Method For Image Compression, Proceedings Of World Academy Of Science, Engineering And Technology Volume 13 May 2006 Issn 1307-6884

[3] K Gopi, Dr. T. Rama Shri, Medical Image Compression Using Wavelets, IOSR Journal of VLSI and Signal Processing (IOSR-JVSP)Volume2, Issue 4(May. - Jun. 2013), PP01-06e-ISSN: 2319-4200, p-ISSN No. : 2319-4197

[4] Mukesh Mittal, Ruchika Lamba, Image Compression Using Vector Quantization Algorithms: A Review, Mittal et al., International Journal of Advanced Research in Computer Science and Software Engineering 3(6), June - 2013, pp. 354-358

[5] Mr. E. PraveenKumar, Dr. M. G. Sumithra, Medical Image Compression Using Integer Multi Wavelets Transform For Telemedicine Applications, International Journal Of Engineering And Computer Science ISSN:2319-7242 Volume 2 Issue 5 May, 2013.

[6] Zixiang Xiong, Kannan Ramchandran, Michael T. Orchard, and Ya-Qin Zhang, A Comparative Study of DCT- and Wavelet-Based Image Coding, Ieee Transactions On Circuits And Systems For Video Technology, Vol. 9, No. 5, August 1999.

[7] Karen Lees "Image Compression Using Wavelets" May 2002. 